\section{Galactofuranose antigens, a target for diagnosis of fungal infections in humans}

The use of biomarkers for the detection of fungal infections is of interest to complement histopathological and culture methods. Since the production of antibodies in immunocompromised patients is scarce, detection of a specific antigen could be effective for early diagnosis. D-Galactofuranose (Galf) is the antigenic epitope in glycoconjugates of several pathogenic fungi. Since Gal $f$ is not biosynthesized by mammals, it is an attractive candidate for diagnosis of infection. A monoclonal antibody that recognizes Galf is commercialized for detection of aspergillosis. The linkage of Galf in the natural glycans and the chemical structures of the synthesized Galf-containing oligosaccharides are described in this paper. The oligosaccharides could be used for the synthesis of artificial carbohydrate-based antigens, not enough exploited for diagnosis.

Lay abstract: D-Galactofuranose (Galf) is the unit in polysaccharides and glycoconjugates of several pathogenic fungi that is recognized by the immune system. Since Galf is not synthesized by mammals, it is an attractive candidate for diagnosis of infection. Since the production of antibodies in immunocompromised patients is scarce, detection of a specific antigen could be effective for early diagnosis. An antibody that recognizes Galf is commercialized for the detection of aspergillosis. Chemically synthesized Galf-containing oligosaccharides, reviewed in this paper, could therefore be used for the synthesis of artificial carbohydrate-based antigens and in diagnosis.

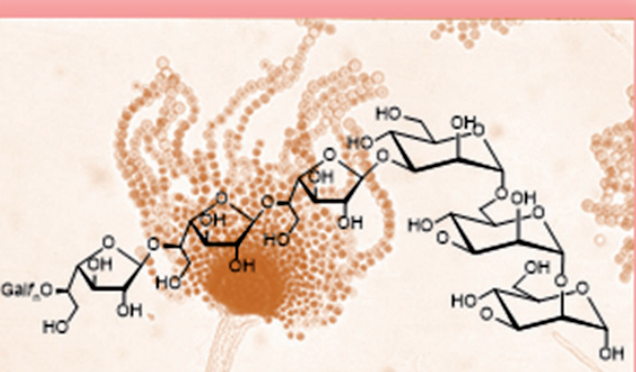

Antigenic structure in Aspergillius glycan

First draft submitted: 7 March 2017; Accepted for publication: 20 March 2017; Published online: 1 June 2017

Keywords: biomarkers $\bullet$ diagnosis $\bullet$ fungal infections $\bullet$ galactofuranose $\bullet$ immune response - synthetic haptens

\section{Carla Marino*,1, Adriana} Rinflerch ${ }^{2}$ \& Rosa M de Lederkremer**,1

'Universidad de Buenos Aires, Consejo Nacional de Investigaciones Científicas y Técnicas, Centro de Investigaciones en Hidratos de Carbono (CIHIDECAR), Departamento de Química Orgánica, Facultad de Ciencias Exactas y Naturales, Pabellón II, Ciudad Universitaria, 1428

Ciudad Autónoma de Buenos Aires, Argentina

${ }^{2}$ Servicio de Dermatología, Dermatología Experimental, Hospital Italiano de Buenos Aires, C1199ACL, Ciudad Autónoma de Buenos Aires, Argentina *Author for correspondence: Tel.: +54 1145763352 cmarino@qo.fcen.uba.ar **Author for correspondence: lederk@qo.fcen.uba.ar 
Effective serodiagnosis of systemic fungal infections is of increasing importance, particularly with regard to the identification of the infective organism. Early diagnosis, before infection is advanced, is still a challenge for efficient therapy. Clinical signs and culture detection methods for diagnosis of fungal infections are often slow, not specific and/or lack sensitivity [1,2]. Although they remain being the usual approaches for diagnosis of mycoses [3], lately, faster, nonculture-based methods have been developed, including immunoassays based on the presence of circulating galactofuranose (Gal $f)$ antigens $[4,5,6,7,8,9]$. Since immunocompromised patients are not capable of producing enough antibodies for their detection in conventional serological tests, monoclonal antibodies have been obtained for the detection of specific antigens [5].

Aspergillosis is the most studied fungus-related disease and thus it will be preferentially referred. Invasive aspergillosis affects the lungs mainly in immunocompromised patients [10]. A review on molecular methods for diagnosis of invasive aspergillosis has been recently published [11].

The monosaccharide $\mathrm{D}$-galactose (D-Gal) is very common in nature as a component of oligosaccharides and glycoconjugates [12]. It is interesting to remark that mammals are only able to synthesize the sugar in the pyranose configuration whereas some bacteria, fungi and protozoa have the unique enzyme UDPGal $p$ mutase (UGM) that catalyzes the conversion of UDP-Galp to UDP-Galf, which is the donor of Galf in the biosynthesis of Galf-containing molecules [13,14]. The presence of Gal $f$ was also reported in some nematodes [15,16]. In fungi, recombinant UGM from Aspergillus fumigatus was described [17]. Studies on the crystal structure and substrate-binding mechanism, revealed differences with the prokaryotic UGM [18]. The synthesis of the substrate UDP-Galp is mediated by a UGE, which catalyzes the interconversion of UDP-Glc and UDP-Gal $p$ [19]. In A. fumigatus, three genes encoding putative UGE have been reported [20], however, only one of them was required for the synthesis of Galf-containing galactomannans and was essential for normal growth. Based on homology, a UGE was identified in $A$. niger [21]. A genetic and transcriptomic analysis of the cell wall of $A$. niger in response to the absence of Gal $f$ was described [22].

Since UDP-Gal $f$ is synthesized in the cytosol [23] and Galf is incorporated in the Golgi, a UDP-Galf transporter is required, and has been identified in A. fumigatus [24]. In this fungus, deletion of UGM caused attenuated virulence of the strain $[18,25]$. The Gal $f$ transferases that incorporate Galf $f$ into the glycans have been studied mainly in Mycobacterium tuberculosis [26,27]. In Aspergillus spp, a Galftransferase involved in the biosynthesis of antigenic $O$-glycans was identified [28]. The glycans that contain Galf are involved in immunological reactions [29,30,31], therefore they are envisioned as targets for diagnosis. This article aims to explore the perspective of methods based on synthetic sugar antigens for the production of monoclonal antibodies for serological detection of fungus-specific antigens.

\section{$\beta$-galactofuranosyl structures in human infective fungi}

Galf is mainly present in the $\beta$-configuration in fungi, many of them involved in mammal infections. It is also present in plant pathogenic fungi, but these will not be discussed in the present review.

Fungi that infect mammals and contain glycans with Gal $f$ are listed in Table 1. Gal $f$ is usually present as terminal sugar, linked to each other by $\beta(1 \rightarrow 6)$ or $\beta(1 \rightarrow 5)$ linkages or branching a mannan core at the $O-2$ or $O-3$ position of mannose.

In 1985, Bennet et al. showed that galactofuranosyl groups are immunodominant in A. fumigatus galactomannan (GM) [45], and accordingly, Galfdeficient mutants of $A$. fumigatus display an attenuated virulence [25].

The cell walls of $A$. fumigatus have at least four different types of molecules containing Gal $f$, which are important for cell wall integrity (Table 1) $[29,32,33,34,35,46,47]$. Gal $f$ units were identified in $O$ - and $N$-linked chains of glycoproteins and also in glycoinositolphosphorylceramides [48]. The glycoinositolphosphorylceramides with $\operatorname{Gal} f(\beta 1 \rightarrow 6)$ linked to a mannose core were highly immunogenic.

Neosartorya spp., teleomorph of A. fumigatus, are a cause of invasive disease in immunocompromised patients [49]. Acute respiratory distress syndrome has been attributed to $N$. udagawae [50]. The structure $[\rightarrow 6)-G a l f(\beta 1 \rightarrow 5)-G a l f(\beta 1 \rightarrow 5)-D-G a l f(\beta 1 \rightarrow]$ has been determined in polysaccharides of some species of Neosartorya [36].

Fusarium is a pathogen of plants, but some species are pathogenic for humans, particularly Fusarium verticillioides and $F$. proliferatum [51]. Fusarium species cause a broad range of opportunistic infections in humans. In healthy individuals, the most common clinical manifestations are onychomycosis, skin infections and keratitis, whereas in immunocompromised patients disseminated infections with multiple necrotic lesions may occur [52].

It is important to discriminate Aspergillus from Fusarium infections since both differ in their response to common antifungals. Using a combination of two antibodies, both species could be differentiated by immunohistology (see below) [31]. 


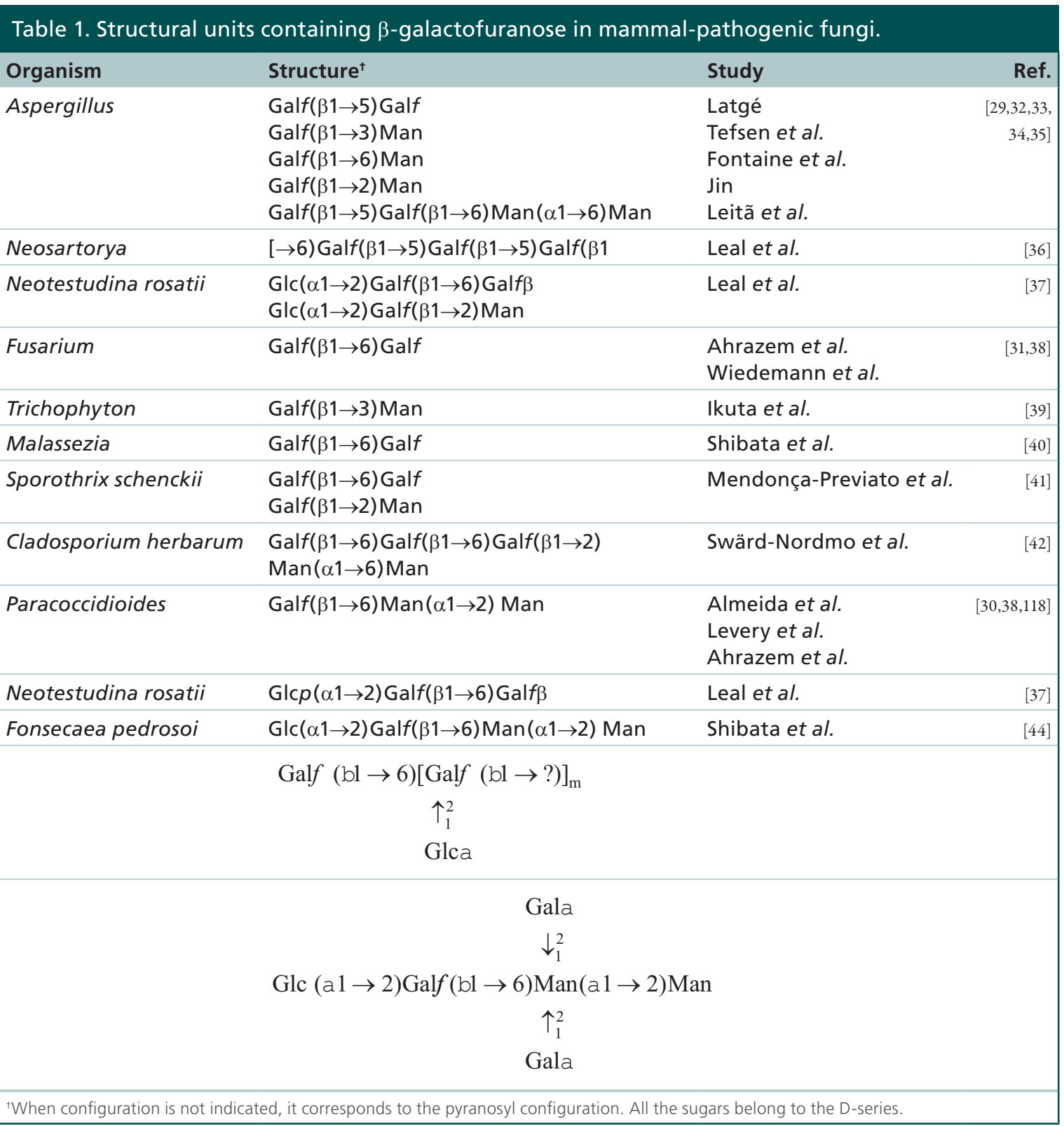

Trichophyton spp., in particular T. mentagrophytes and $T$. rubrum cause chronic dermatophyte infections, often associated with infection of the nails (onychomycosis) [53]. Although they first infected animals, they adapted to infect humans and are now considered a major health problem [54]. Cell wall antigens secreted by the fungus may diffuse into the dermis and establish the infection, due to immunosuppressive effects. Impairment of lymphocyte proliferation was shown [55]. One of the major cell wall components secreted to the medium is a GM. Structure determinations showed that Gal $f$ terminal units are $\beta(1 \rightarrow 3)$ linked to a mannan core. Polygalactofuranosyl chains, similar to those produced by
A. fumigatus, have not been found in Trichophyton [39]. Accordingly, a monoclonal antibody against the GM of $A$. fumigatus showed very low cross-reactivity with exoantigens from cultures obtained from clinical specimens [56]. Glycosylphosphatidylinositols labeled with $\left[{ }^{3} \mathrm{H}\right]$-galactose and $\left[{ }^{3} \mathrm{H}\right]$-mannose were biosynthesized by membrane preparations of $T$. rubrum. The lability to acid of the galactose suggested its furanosic configuration [57].

A linear chain of $\beta 1 \rightarrow 6$ linked Galf attached to a small mannan was also found in polysaccharides of Malassezia spp [40]. Malassezia spp. are human pathogens responsible for skin diseases and they are also associated with catheter-related fungaemia [58]. 
Sporothrix schenckii is the agent of sporotrichosis in humans and animals, producing skin and subcutaneous lesions. It is present in all continents, especially in tropical and subtropical areas [59,60]. In an early work, Galf-containing polysaccharides were isolated from the supernatants of $S$. schenckii cultures [41]. It was found that a mannan core is substituted by $\beta-G a l f$ chains which are responsible for cross-reactions with other fungal antigens. Apparently, no further studies on this GM were described. In turn, peptidorhamnomannans with both $N$ - and $O$-linked carbohydrate chains as the immunodominant structures were obtained from extracts of the cell walls [61]. Later studies characterized a peptidorhamnomannan (Gp70) isolated from the yeast phase of $S$. schenckii as an adhesin involved in the host-pathogen interaction, but Gal $f$ was not reported as a constituent of this glycoprotein [62].

Cladosporium spp. are mainly plant pathogens but some may trigger allergic reactions in sensitive individuals. Prolonged exposure to a high concentration of spores may produce chronic asthma. A glycoprotein named Ag-54, which contains $80 \%$ carbohydrate, was an allergen purified from $C$. herbarum. Structural studies showed chains of $\beta 1 \rightarrow 6$ linked Gal $f$ bound to $O-2$ of $1 \rightarrow 6$ linked mannoses (Table 1) [42].

Paracoccidioides brasiliensis is endemic to regions of Latin America, causing a mycosis which spreads from the lungs to many organs and if not treated could be fatal [63]. Paracoccidioidomycosis (PCM) is commonly diagnosed by identifying budding yeast cells in biological fluids or histologically [64]. Glycolipids extracted from yeast and mycelium forms of $P$. brasiliensis reacted with sera from patients. The antibodies were directed mainly to the galactofuranosyl units in the glycolipids [65]. A review on glycolipids in fungi and trypanosomatids discusses the role of Galf in the infectivity [66]. However, the main diagnostic antigen of $P$. brasiliensis is the exocellularly secreted glycoprotein gp 43 , which contains a terminal Gal $f$ unit attached $(\beta 1 \rightarrow 6)$ to a mannose in an $N$-linked oligosaccharide chain [30].

Antigenic Gal $f$ is usually present as an exposed terminal sugar in the glycan (Table 1). However, in some fungi an internal Gal $f$ is present. This is the case of Neotestudina rosatii, from which three polysaccharides containing the units $\operatorname{Glc}(\alpha 1 \rightarrow 2) \mathrm{Gal} f(\beta 1 \rightarrow 6) \mathrm{Gal} f-$ and $\operatorname{Glc}(\alpha 1 \rightarrow 2) \operatorname{Gal} f(\beta 1 \rightarrow 2)$ Man linked to a mannan were extracted [37].

Neotestudina rosatii is one of the etiologic agents of subcutaneous infections in humans. This species occurs with other fungi causing eumycetoma, and its taxonomic classification is uncertain [67].

A glycoprotein with $N$ - and $O$-glycans was extracted from Fonsecaea pedrosoi, the etiologic agent of chromoblastomycosis. The hexasaccharide containing internal Gal $f$ (Table 1) was the main $O$-linked chain $[44,68]$. Specific antibodies against $F$. pedrosoi were strongly inhibited by the hexose, but the contribution of the internal Gal $f$ to the antigenicity was not evaluated. Probably, the main epitope in F. pedroso $i$ is the external $\alpha-G a l p$, a recognized antibody in humans [69].

In the previously mentioned examples, Gal $f$ is present in the $\beta$-anomeric configuration. However, the less common $\alpha$-Gal $f$ has also been found in some organisms, such as, P. brasiliensis. Interestingly, the configuration of the sugar depends on the phase, whereas $\beta-\mathrm{Gal} f$ is present in the yeast form infecting the mammal, $\alpha-\mathrm{Gal} f$ is a constituent of the mycelial GM (Table 2) [38,70].

Galf was also found in glycoinositolphospholipids obtained from the yeast phase of Histoplasma capsulatum, the causative agent of histoplasmosis, an endemic mycosis [71]. Histoplasma capsulatum is a dimorphic fungus which grows in the soil as a filamentous mycelium, but it converts to a yeast-like form in the tissues of infected animals. The purified glycoinositolphospholipids were shown to react with sera from histoplasmosis patients [71]. The authors tentatively assigned the $\alpha$-configuration for the Gal $f$ from the yeast form, in contrast with the configuration found for the Galf in the same phase in P. brasiliensis. The configuration of galactofuranosides is unequivocally assigned by NMR spectroscopy [12], which was not used by Barr et al. in 1984 [71]. The glycosphingolipids from the mycelial phase were not described.

In $A$. niger, $\alpha-G a l f(1 \rightarrow 2)$ linked to mannose was characterized [72]. Although this species is less pathogenic for humans than $A$. fumigatus, it could produce allergic reactions in high concentrations.

Table 2. Structural units containing $\alpha$-galactofuranose in mammal-pathogenic fungi.

\begin{tabular}{|lllr|}
\hline $\begin{array}{l}\text { Organism } \\
\text { Paracoccidioides brasiliensis }\end{array}$ & Structure $^{\dagger}$ & Study & Ref. \\
& Galf $(\alpha 1 \rightarrow 6)$ Man $(\alpha 1 \rightarrow 2)$ Man & $\begin{array}{l}\text { Ahrazem et al. } \\
\text { San-Blas et al. }\end{array}$ & {$[38,70]$} \\
\hline Histoplasma capsulatum & Galf $(\alpha 1 \rightarrow 6)$ Man $(\alpha 1 \rightarrow 2$ or 6$)$ & Barr et al. & {$[71]$} \\
\hline Aspergillus niger & Galf $(\alpha 1 \rightarrow 2)$ Man & Takayanagi et al. & {$[72]$} \\
\hline${ }^{+}$All the sugars belong to the D-series. & & & \\
\hline
\end{tabular}




\section{Table 3. Structures with galactofuranose units found in fungal glycans which have been chemically synthesized.}

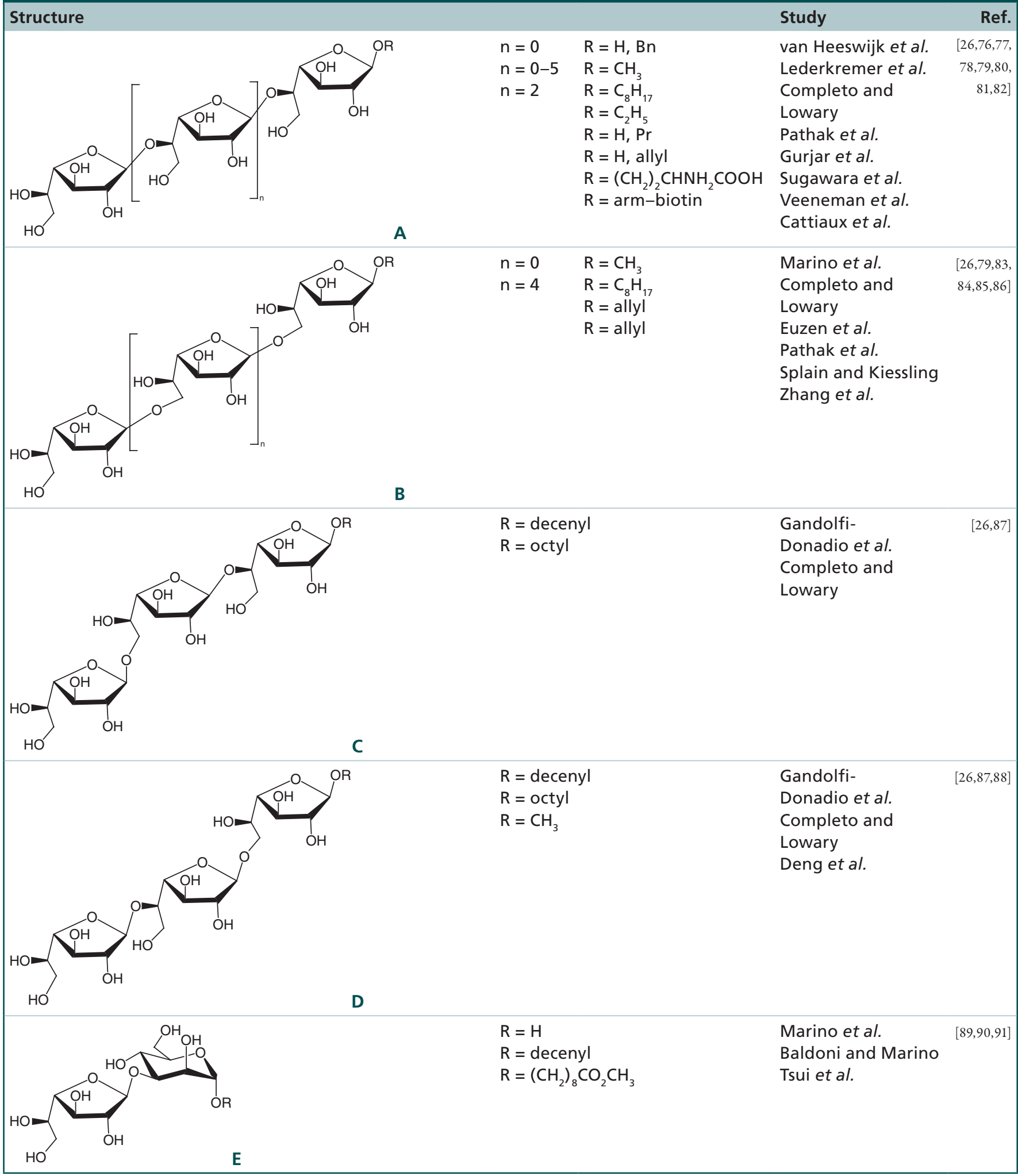


Table 3. Structures with galactofuranose units found in fungal glycans which have been chemically synthesized (cont.).

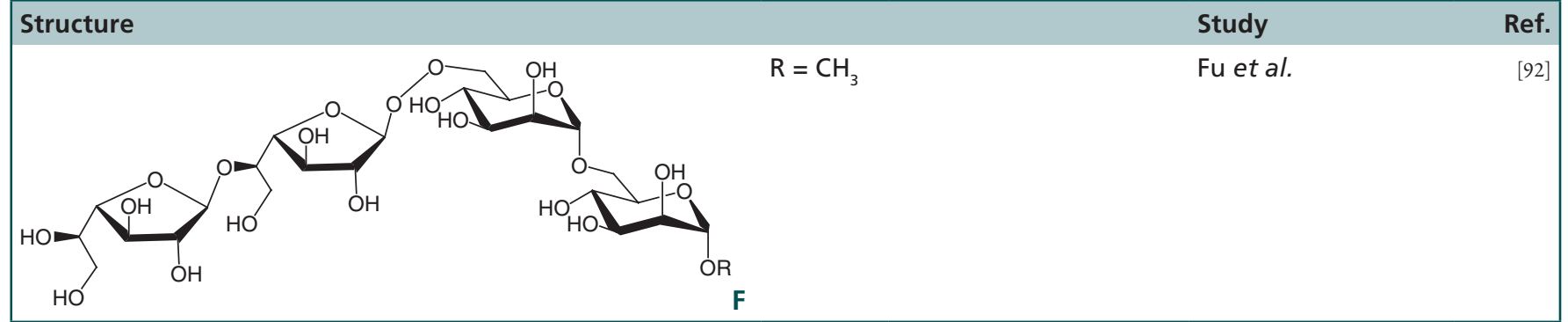

The antigenic properties of $\beta$-galactofuranosides are well known $[29,30,31]$ but the immunological role of $\alpha$-Gal $f$ was apparently not described. The differences in the Gal $f$ configuration in the galactomannans of the infective $P$. brasiliensis and $A$. fumigatus could explain the low cross-reactivity in serological tests using a specific antibody [73].

\section{Structural analysis of Galf-containing polysaccharides or glycoconjugates}

In earlier works, the identification of Gal $f$ units in glycoconjugates was performed using chemical methods. Taking advantage of the greater lability of furanosic linkages with respect to the pyranosic bonds, terminal Gal $f$ units may be selectively released from the molecule by mild acid hydrolysis, separated by chromatographic techniques or by dialysis, depending on the MW of the remaining degraded molecule, and then analyzed by chromatographic methods or even GC-MS. Also, the exocyclic chain of terminal Gal $f$ could be selectively oxidized by periodate under mild conditions, and upon reduction with $\mathrm{NaBH}_{4}$ converted to arabinofuranose, which may be identified after acid hydrolysis by GC-MS. Application of these methods is exemplified in the report on the determination of the structure of glycolipids of $P$. brasiliensis [65]. Information on the substitution position of the galactofuranosyl linkages is provided by methylation analysis [74].

NMR has also been useful for the identification of Gal $f$ units in oligosaccharides. ${ }^{1} \mathrm{H}$ NMR spectra usually show deshielded anomeric signals for Galf units, compared with those of pyranosic units. Particularly useful is the ${ }^{13} \mathrm{C}$ NMR spectroscopy because the furanosyl ring has a specific signal pattern, easily distinguishable from the pyranosyl analog. For $\beta$-Galf units, signals corresponding to the anomeric carbon, and $\mathrm{C}-2$ and $\mathrm{C}-4$ resonate significantly deshielded in comparison with pyranosic signals. In addition, $\alpha$-Gal $f$ has also a particular pattern. Advances in NMR spectroscopy have facilitated the characterization of oligo- saccharides. Thus, bidimensional techniques allow not only the identification of the monosaccharides and their anomeric configuration but also to stablish the position of substitution and sequences [35,39,75].

\section{Chemically synthesized oligosaccharides of antigenic glycans}

The use of chemically synthesized oligosaccharides of defined structure avoids the disadvantages of glycans obtained from natural sources, such as, the difficulty of isolating and purificating sufficient quantities, and problems associated with their heterogeneity. Also, more specificity may be achieved by using a neoantigen. Following this line, a biotinylated tetrasaccharide with $\operatorname{Gal} f(\beta 1 \rightarrow 5) \mathrm{Gal} f$ linkages was synthesized (Table $3 A ; n=2)$. This synthetic antigen was recognized by the monoclonal antibody EB-A2 against Aspergillus [76].

In Table 3, the structures of Galf-containing oligosaccharides, which have been chemically synthesized and are part of fungal glycans, are shown. Most efforts have been directed to the synthesis of oligosaccharides containing Gal $f(\beta 1 \rightarrow 5) \mathrm{Gal} f$, the antigenic unit in Aspergillus $[26,77,78,79,80,81,82]$.

Several chemical syntheses for the Gal $f(\beta 1 \rightarrow 6)$ Gal $f$ disaccharide have been reported (Table 3B) $[26,83,84,85]$. This is the minimal epitope in Fusarium glycans [31]. A hexasaccharide with the same linkages was synthesized [86]. The two trisaccharides containing both types of linkages (Table 3C \& D) were prepared [26,87,88] because of their importance as repeating units in the arabinogalactan of M. tuberculosis [93], but is also present in a polysaccharide extracted from Neosartorya [36]. Galf is frequently linked to mannose forming galactomannans. The synthesis of the disaccharide Gal $f(\beta 1 \rightarrow 3)$ Man (Table 3E) $[89,90,91]$ and the more complex heterotetrasaccharide (Figure 1F) [92], which was indicated as the minimal epitope in the mycelial cell wall of $A$. fumigatus [35], were described.

The synthesis of Galf-containing molecules requires the efficient preparation of derivatives of 
D-Gal in the furanosic configuration, free from the pyranosic forms, as precursors of Galf units in the target molecules. Furthermore, efficient glycosylation methodologies and the consequent availability of galactofuranosyl donors as well as conveniently substituted derivatives as glycosyl acceptors are required (Figure 1) [27,94,95,96].

Most sugars lead by conventional methods to pyranosic derivatives, which are thermodynamically more stable. However, in conditions under which other monosaccharides lead mainly to pyranosic products, large proportions of galactofuranosic derivatives can be obtained from galactose. This is the case of the traditional Fisher glycosylation, which yields a mixture of furanosic and pyranosic glycosides that must be separated by chromatography [27]. An example may be found in the preparation of the pentenyl galactofuranosides which were used as precursors in glycosylation reactions [97].

The peracylated derivatives are commonly used as precursors, particularly penta-O-benzoyl-Galf $(\mathrm{BzGal} f$ ) that may be obtained as crystalline products, in one step, from galactose [98]. Glycosylation of $\mathrm{BzGal} f$ promoted by a Lewis acid affords the $\beta$-galactofuranosides, as a result of anchimeric assistance. In Figure 2, an example for the synthesis of the natural disaccharides $\operatorname{Gal} f(\beta 1 \rightarrow 5) \mathrm{Gal} f$ [83] and $\mathrm{Gal} f(\beta 1 \rightarrow 6) \mathrm{Gal} f[78]$ is shown.

One of the most popular glycosylation procedures is the trichloroacetimidate method [99], which is compatible with acid-sensitive acceptors and was used first for the synthesis of $\operatorname{Gal} f(\beta 1 \rightarrow 3)$ GlcNAc [100]. As this is an area of active research, several more methods have been designed and extensively reviewed $[26,95,96]$. The synthetic oligosaccharides could be precursors for artificial antigens to be used for diagnosis [101] or for the construction of synthetic vaccines [102,103].

\section{Immunologic detection of Galf-containing molecules}

A monoclonal antibody, which recognized $\mathrm{Gal} f(\beta 1 \rightarrow 5) \mathrm{Gal} f$ epitopes was produced by immunizing rats with an extract of $A$. fumigatus mycelia. The antibody called EB-A2 also detected the GM of other Aspergillus species, using an ELISA assay [104,105]. It was commercialized as Platelia ${ }^{\mathrm{TM}}$ Aspergillus ELISA (Bio-Rad Laboratories and Sanofi Diagnostics, CA, USA). The specificity and sensitivity of the assay were reviewed [106]. Multivalent gold nanoparticles carrying Gal $f$ were recognized by this antibody [107] . The presence of GM in circulation depends on the manifestation of the disease. For instance, negative results are commonly obtained for the detection of GM in sera of allergic bronchopulmonary aspergillosis. Reviews on diagnosis of aspergillosis have been published [108,109,110]. Apparently, this is the only commercialized test based on detection of Galf. It was shown that EB-A2 detects GM in supernatants of several Fusarium species [111,112]. However, Wiedemann et al. described a novel monoclonal antibody, AB 135-8, which recognizes Galfin Fusarium but as part of a different antigen [31]. In fact, the structural unit Gal $\beta(1 \rightarrow 6) \mathrm{Gal} f$, was characterized in a polysaccharide extracted from Fusarium [75] but it was not reported in Aspergillus (Table 1). The same antigenic disaccharide was found in Malassezia galactomannans, which did not react with the EB-A2 antibody against $A$. fumigatus, but apparently was not tested with AB 135-8 against Fusarium. Antibodies against M. furfur did not react with galactomannans of A. fumigatus, $T$. rubrum or $F$. pedrosoi, confirming the presence of a different linkage for the Gal $f$ units, but apparently these were not tested with $\mathrm{AB}$ 135-8 against Fusarium [40].

The Platelia test was negative for yeast and mold forms of S. schenckii [113]. Controversial results were

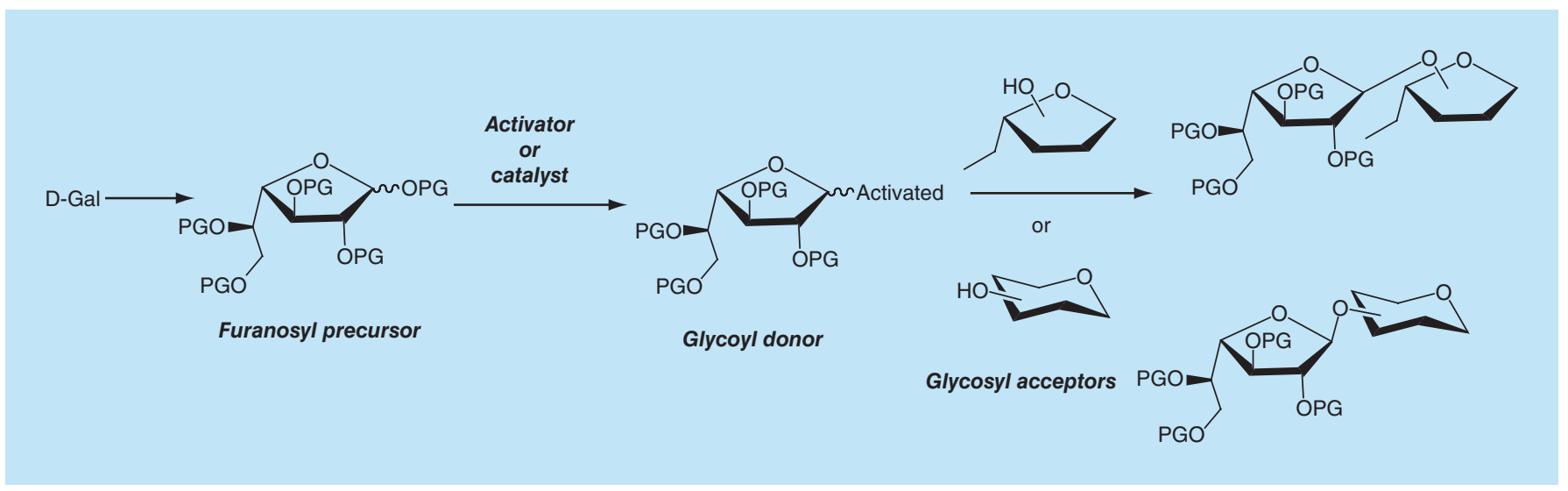

Figure 1. General strategy for the synthesis of Galf-containing molecules. 


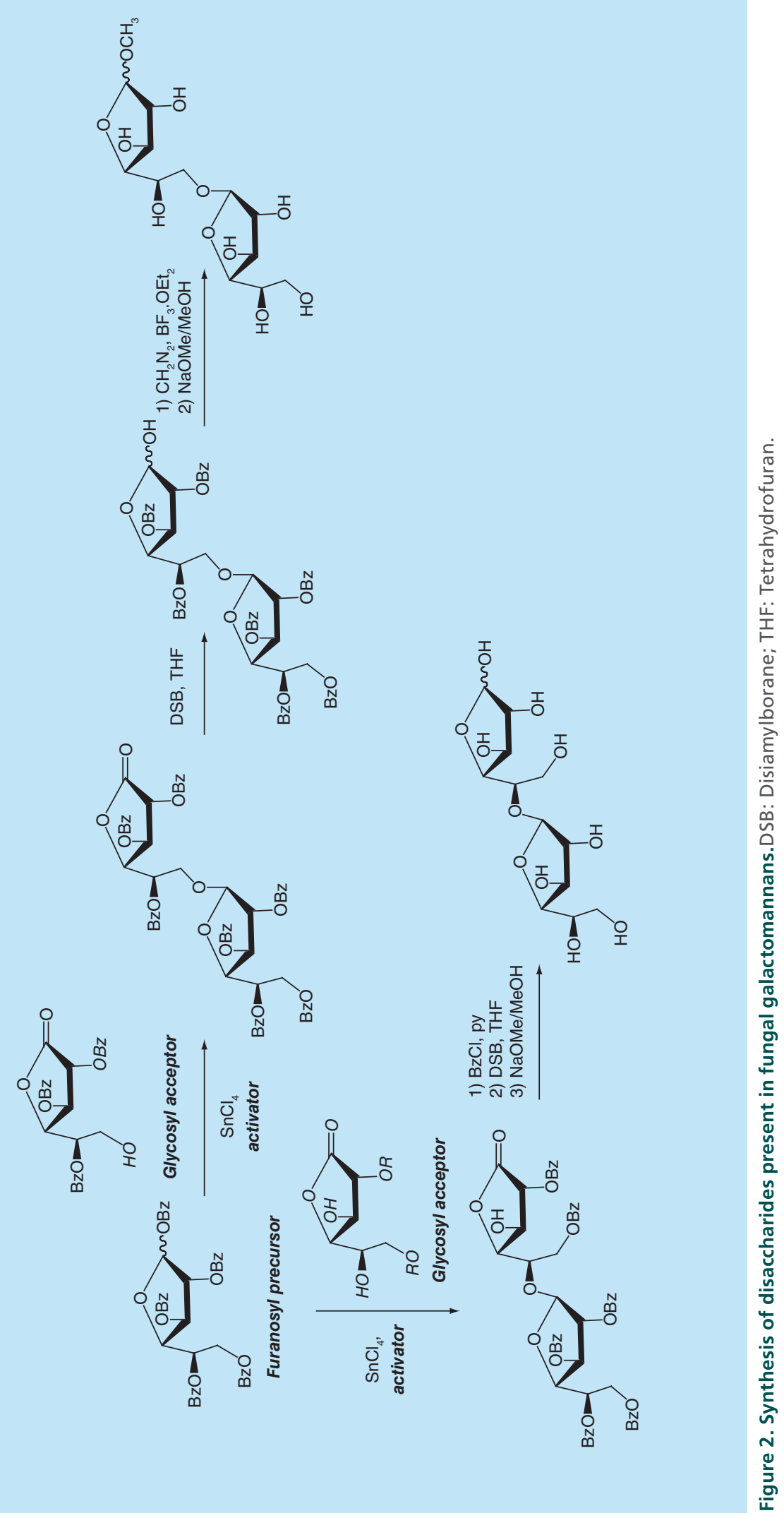


reported for Cryptococcus neoformans. While a positive test was reported for a patient with $C$. meningitis [114], a later report revealed no cross-reaction with culture extracts, purified polysaccharides, clinical specimens and specimens from animals following experimental infection [115]. These results are explained by the composition of the cell wall of C. neoformans. Although galactoxylomannan is a constituent of the cell wall, and D-Gal is its major component, this sugar is mainly in the pyranosic configuration, with only $2 \%$ tentatively assigned to Galf, considering a small peak in the ${ }^{13} \mathrm{C}$ NMR spectrum [116], which is as explained above, is not enough to guarantee the configuration. More recently, it was demonstrated that Gal $f$ is not required for growth or virulence of $C$. neoformans [117].

In $P$. brasiliensis, $\mathrm{Gal} f$ is the immunodominant unit in a glycosylinositolphosphoryl ceramide [43], however, the main diagnostic antigen is the glycoprotein gp 43 . Studies of $\mathrm{N}$-deglycosylation gave rise to a protein of $38 \mathrm{kDa}$, which strongly reacted with sera from patients with PCM [118]. Also, recombinant gp 43 isoforms as $N$-mannosylated proteins were expressed in the yeast Pichia pastoris and showed good specificity for detection of PCM in sera [119]. These results indicate the presence of other epitopes in gp 43, although the cause of cross-reactivity with sera from patients with other mycoses would be the terminal Gal $f$ [118120]. Immunodiagnosis of gp43 in PCM using a latex test was recently described [73].

In the previous examples, Gal $f$ is present in the galactomannans in the $\beta$-anomeric configuration. A few examples of $\alpha$-Gal $f$ may be seen in Table 2. The antigenic properties of $\alpha$-Gal $f$ were apparently not described. As expected, isolates from $H$. capsulatum in yeast and mold phases were not recognized by the monoclonal antibody against the Aspergillus GM [113]. However, it was reported that some patients with dis- seminated histoplasmosis gave positive results with this antibody [121].

\section{Conclusion \& future perspective}

Gal $f$ is an antigenic structural unit in many infectious fungi, which is not biosynthesized by humans. The preparation of synthetic neoglycoconjugates containing $\beta$-Gal $f$ for the diagnosis of some extended mycoses as aspergillosis is a field worth to explore. The synthetic antigens would help define the structure of the corresponding epitopes and in a more ambitious project they could be the starting line for the synthesis of carbohydrate-based vaccines [102]. In reviewing the natural structures, it is challenging to understand why in some dimorphic fungi the commonly found $\beta$-Gal $f$ changes the anomeric configuration to $\alpha-G a l f$, in the transition between phases. The $\alpha$-Gal $f$ transferases necessary for the construction of the linkages in some galactomannans have not yet been described.

\section{Authors' contributions}

C Marino and RM de Lederkremer contributed with the design, acquisition and interpretation of data, writing and revision. A Rinflerch provided her expertise in medical aspects.

\section{Financial \& competing interests disclosure}

The authors are indebted to the University of Buenos Aires (UBA), the National Research Council of Argentina (CONI(ET) and the National Agency for Promotion of Science and Technology (ANPCyT). C Marino and RM de Lederkremer are research members of CONICET. The authors have no other relevant affiliations or financial involvement with any organization or entity with a financial interest in or financial conflict with the subject matter or materials discussed in the manuscript apart from those disclosed.

No writing assistance was utilized in the production of this manuscript.

\section{Executive summary}

\section{Biomarkers}

- Diagnosis of fungal infections and identification of the causative agent is important for the selection of an accurate therapy. The use of biomarkers may provide faster results and complement culture and histological methods.

\section{Carbohydrate-based markers}

- Galactofuranose (Galf) is an attractive candidate as a biomarker for diagnosis of infections, since it is the antigenic epitope in glycans of several pathogenic fungi and is not biosynthesized by mammals. An ELISA assay based on the EB-A2 antibody that detects the Galf in the GM of Aspergillus is commercialized. The different linkages of the Galf to other sugars in the fungal carbohydrates may provide specificity, a line of research that needs further studies.

Chemically synthesized oligosaccharides of antigenic glycans

- The impressive advance achieved in the chemical synthesis of the natural oligosaccharides encourages their use as specific antigens. 
Open access

This work is licensed under the Creative Commons Attribution

\section{References}

Papers of special note have been highlighted as: $\bullet$ of interest;

• of considerable interest

1 Einsele H, Hebart H, Roller G et al. Detection and identification of fungal pathogens in blood by using molecular probes. J. Clin. Microbiol. 35, 1353-1360 (1997).

2 Erjavec $Z$, Verweij PE. Recent progress in the diagnosis of fungal infections in the immunocompromised host. Drug Resist. Updates 5, 3-10 (2002).

3 Lu Y-Y, Hong C-H, Lee C-H. Laboratory techniques used in the diagnosis of mycosis. www.esciencecentral.org/

4 Reiss E, Obayashi T, Orle K, Yoshida M, Zancope-Oliveir RM. Non-culture based diagnostic tests for mycotic infections. Med. Mycol. 38(Suppl. 1), 147-159 (2002).

5 Alexander B, Pfaller M. Contemporary tools for the diagnosis and management of invasive mycoses. Clin. Infect. Dis. 43, S15-S27 (2006).

- A review on diagnostic technologies and their clinical application.

6 Ostrosky-Zeichner L. Invasive mycoses: diagnostic challenges. Am. J. Med. 125, S14-S24 (2012).

7 O'Shaughnessy EM, Shea YM, Witebsky FG. Laboratory diagnosis of invasive mycoses. Infect. Dis. Clin. North. Am. 17, 135-158 (2003).

8 Lau A, Chen S, Sleiman S, Sorrell T. Current status and future perspectives on molecular and serological methods in diagnostic mycology. Future Microbiol. 4, 1185-222 (2009).

9 Pfaller MA. Invasive fungal infections and approaches to their diagnosis. Methods Microbiol. 42, 219-287 (2015).

10 Maschmeyer G, Haas A, Cornely OA. Invasive aspergillosis: epidemiology, diagnosis and management in immunocompromised patients. Drugs 67, 1567-1601 (2007).

11 Ambasta A, Carson J, Church DL. The use of biomarkers and molecular methods for the earlier diagnosis of invasive aspergillosis in immunocompromised patients. Med. Mycol. 53, 531-557 (2015).

12 Marino C, Lederkremer RM. Galactose configurations in nature with emphasis on the biosynthesis of galactofuranose in glycans. In: Galactose: Structure and Function in Biology and Medicine (Vol 2). Pomin VH (Ed.). Nova Science Publisher, NY, USA, 107-133 (2014).

13 Partha SK, van Straaten KE, Sanders DAR. Structural basis of substrate binding to UDP-galactopyranose mutase: crystal structures in the reduced and oxidized state complexed with UDP-galactopyranose and UDP. J. Mol. Biol. 394, 864-877 (2009).

14 Partha SK, Sadeghi-Khomami A, Slowsk K et al. Chemoenzymatic synthesis, inhibition studies and X-ray crystallographic analysis of the phosphono analogue of UDP-Gal $p$ as an inhibitor and mechanistic probe for
4.0 License. To view a copy of this license, visit http://creativecommons.org/licenses/by/4.0/

UDP-galactopyranose mutase. J. Mol. Biol. 403, 578-590 (2010).

15 Novelli JF, Chaudhary K, Canovas J et al. Characterization of the Caenorhabditis elegans UDP-galactopyranose mutase homolog glf-1 reveals an essential role for galactofuranose metabolism in nematode surface coat synthesis. Dev. Biol. 335, 340-355 (2009).

16 Misra S, Valicherla GR, Shahab M et al. UDPGalactopyranose mutase, a potential drug target against human pathogenic nematode Brugia malayi. Pathog. Dis. 74, pii:ftw072 (2016) (Epub ahead of print).

17 Oppenheimer M, Poulin MB, Lowary TL, Helm RF, Sobrado P. Characterization of recombinant UDPgalactopyranose mutase from Aspergillus fumigatus. Arch. Biochem. Biophys. 502, 31-38 (2010).

18 van Straaten KE, Routier FH, Sanders DAR. Structural insight into the unique substrate binding mechanism and flavin redox state of UDP-galactopyranose mutase from Aspergillus fumigatus. J. Biol. Chem. 287, 10780-10790 (2012).

19 Holden HM, Rayment I, Thoden JB. Structure and function of enzymes of the Leloir pathway for galactose metabolism. J. Biol. Chem. 278, 43885-43888 (2003).

20 Lee MJ, Gravelat FN, Cerone RP et al. Overlapping and distinct roles of Aspergillus fumigatus UDP-glucose 4-epimerases in galactose metabolism and the synthesis of galactose-containing cell wall polysaccharides. J. Biol. Chem. 289, 1243-1256 (2014).

- A genetic study to understand the role of enzymes involved in the biosynthesis of the cell wall of Aspergillus.

21 Park J, Tefsen B, Arentshorst M et al. Identification of the UDP-glucose-4-epimerase required for galactofuranose biosynthesis and galactose metabolism in A. niger. Fungal Biol. Biotechnol. 1, 6 (2014).

22 Park J, Hulsman M, Arentshorst M et al. Transcriptomic and molecular genetic analysis of the cell wall salvage response of Aspergillus niger to the absence of galactofuranose synthesis. Cell. Microbiol. 18, 1268-1284 (2016).

23 Kleczka B, Lamerz AC, van Zandbergen G et al. Targeted gene deletion of Leishmania major UDP-galactopyranose mutase leads to attenuated virulence. J. Biol. Chem. 282, 10498-10505 (2007).

24 Engel J, Schmalhorst PS, Dork-Bousset T, Ferrieres V, Routier FH. A single UDP-galactofuranose transporter is required for galactofuranosylation in Aspergillus fumigatus. J. Biol. Chem. 284, 33859-33868 (2009).

25 Schmalhorst P, Krappmann S, Vervecken W et al. Contribution of galactofuranose to the virulence of the opportunistic pathogen Aspergillus fumigatus. Eukaryot. Cell 7, 1268-1277 (2008).

-. The contribution of galactofuranose (D-Galf) to the virulence of $A$. fumigatus is shown by deletion of a gene that controls its biosynthesis. 
26 Completo GC, Lowary TL. Synthesis of galactofuranosecontaining acceptor substrates for mycobacterial galactofuranosyltransferases. J. Org. Chem. 73, 4513-4525 (2008).

27 Marino C, Gallo-Rodriguez C, Lederkremer RM. Galactofuranosyl-containing glycans: Occurrence, synthesis, and biochemistry. In: Glycans: Biochemistry, Characterization and Applications. Mora-Montes HM (Ed.). Nova Science Publishers, NY, USA, 207-268 (2012).

- A comprehensive review on the occurrence of galactofuranosides in nature and their chemical synthesis.

28 Komachi Y, Hatakeyama S, Motomatsu $\mathrm{H}$ et al. gfsA encodes $\mathrm{K}$ a novel galactofuranosyltransferase involved in biosynthesis of galactofuranose antigen of $O$-glycan in Aspergillus nidulans and A. fumigatus. Mol. Microbiol. 90, 1054-1073 (2013).

29 Latgé JP. Galactofuranose containing molecules in Aspergillus fumigatus. Med. Mycol. 47, S104-S109 (2009).

- Discusses the presence of D-Gal $f$ containing glycoconjugates in A. fumigatus.

30 Almeida IC, Neville DCA, Mehlert A et al. Structure of the $\mathrm{N}$-linked oligosaccharide of the main diagnostic antigen of the pathogenic fungus Paracoccidioides brasiliensis. Glycobiology 6, 507-515 (1996).

31 Wiedemann A, Kakoschke TK, Speth C et al. Distinct galactofuranose antigens in the cell wall and culture supernatants as a means to differentiate Fusarium from Aspergillus species. Int. J. Med. Microbiol. 306, 381-390 (2016).

32 Tefsen B, Ram AF, van Die I, Routier FH. Galactofuranose in eukaryotes: aspects of biosynthesis and functional impact. Glycobiology 22, 456-469 (2012).

- A review highlighting recent advances in the understanding of the biosynthesis and function of Galf-containing glycoconjugates in Aspergillus spp. and trypanosomatid parasites.

33 Fontaine T, Delangle A, Simenel C et al. Galactosaminogalactan, a new immunosuppressive polysaccharide of Aspergillus fumigatus. PLoS Pathog. 7, e1002372 (2012).

34 Jin C. Protein glycosylation in Aspergillus fumigatus is essential for cell wall synthesis and serves as a promising model of multicellular eukaryotic development. Int. J. Microbiol. 2012, 654251 (2012).

35 Leitã EA, Bittencourt VC, Haido RM et al. Betagalactofuranose-containing $O$-linked oligosaccharides present in the cell wall peptidogalactomannan of Aspergillus fumigatus contain immunodominant epitopes. Glycobiology 13, 681-692 (2013).

36 Leal JA, Jiménez-Barbero J, Gómez-Miranda B et al. Structural investigations of cell wall polysaccharides from Neosartorya. Relationships with their putative anamorphs of Aspergillus. Carbohydr. Res. 273, 255-262 (1995).

37 Leal JA, Giménez-Abián MI, Canales Á et al. Cell wall polysaccharides isolated from the fungus Neotestudina rosatii, one of the etiologic agents of mycetoma in man. Glycoconj. J. 26, 1047-1054 (2009).
38 Ahrazem O, Prieto A, San-Blas G et al. Structural differences between the alkali-extracted water-soluble cell wall polysaccharides from mycelial and yeast phases of the pathogenic dimorphic fungus Paracoccidioides brasiliensis. Glycobiology 13, 743-747 (2003).

- Describes different anomeric configurations for the D-Galf in the yeast and mycelial phases of Paracoccidioides brasiliensis.

39 Ikuta K, Shibata N, Blake JS et al. NMR study of the galactomannans of Trichophyton mentagrophytes and Trichophyton rubrum. Biochem. J. 323, 297-305 (1997).

40 Shibata N, Saitoh T, Tadokoro Y, Okawa Y. The cell wall galactomannan antigen from Malassezia furfur and Malassezia pachydermatis contains beta-1,6-linked linear galactofuranosyl residues and its detection has diagnostic potential. Microbiology 155, 3420-3429 (2009).

41 Mendonça-Previato L, Gorin PA, Travassos LR. Galactosecontaining polysaccharides from the human pathogens Sporothrix schenckii and Ceratocystis stenoceras. Infect. Immun. 29, 934-939 (1980).

42 Swärd-Nordmo M, Paulsen BS, Wold JK. The glycoprotein allergen Ag-54 (Cla h II) from Cladosporium herbarum. Structural studies of the carbohydrate moiety. Int. Arch. Allergy Appl. Immunol. 85, 288-294 (1988).

43 Albuquerque CF, Marques da Silva SH, Camargo ZP. Improvement of the specificity of an enzyme-linked immunosorbent assay for diagnosis of paracoccidioidomycosis. J. Clin. Microbiol. 43, 1944-1946 (2005).

44 Shibata N, Okawa Y. Chemical structure of betagalactofuranose-containing polysaccharide and O-linked oligosaccharides obtained from the cell wall of pathogenic dematiaceous fungus Fonsecaea pedrosoi. Glycobiology 21, 69-81 (2011).

45 Bennett JE, Bhattacharjee AK, Glaudemans CPJ. Galactofuranosyl groups are immunodominant in Aspergillus fumigatus galactomannan. Mol. Immunol. 22, 251-254 (1985).

46 Latgé JP, Kobayashi H, Debeaupuis JP et al. Chemical and immunological characterization of the extracellular galactomannan of Aspergillus fumigatus. Infect. Immun. 62, 5424-5433 (1994).

47 Morelle W, Bernard M, Debeaupuis J-P, Buitrago M, Tabouret M, Latgé J-P. Galactomannoproteins of Aspergillus fumigatus. Eukaryot. Cell 4, 1308-1316 (2005).

48 Toledo MS, Levery SB, Bennion B et al. Analysis of glycosylinositol phosphorylceramides expressed by the opportunistic mycopathogen Aspergillus fumigatus. J. Lipid Res. 48, 1801-1824 (2007).

49 Balajee SA, Gribskov J, Brandt M et al. Mistaken identity: Neosartorya pseudofischeri and its anamorph masquerading as Aspergillus fumigatus. J. Clin. Microbiol. 43, 5996-5999 (2005).

50 Farrell JJ, Kasper DJ, Taneja D et al. Acute respiratory distress caused by Neosartorya udagawae. Med. Mycol. Case Rep. 6, 1-5 (2014).

51 Nucci M, Anaissie E. Fusarium infections in immunocompromised patients. Clin. Microbiol. Rev. 20, 695-704 (2007). 
52 van Diepeningen AD, Brankovics B, Iltes J, van der Lee TA, Waalwijk C. Diagnosis of Fusarium infections: approaches to Identification by the clinical mycology laboratory. Curr. Fungal Infect. Rep. 9, 135-143 (2015).

53 Hay RJ. Chronic dermatophyte infections. I. Clinical and mycological features. Br. J. Dermatol. 106, 1-7 (1982).

54 Woodfolk JA. Allergy and dermatophytes. Clin. Microbiol. Rev. 18, 30-43 (2005).

55 Waldman A, Segal R, Berdicevsky I, Gilhar A. CD4 ${ }^{+}$ and $\mathrm{CD} 8^{+} \mathrm{T}$ cells mediated direct cytotoxic effect against Trichophyton rubrum and Trichophyton mentagrophytes. Int. J. Dermatol. 49, 149-157 (2010).

56 Swannink CM, Meis JF, Rijs AJ, Donnelly JP, Verweij PE. Specificity of a sandwich enzyme-linked immunosorbent assay for detecting Aspergillus galactomannan. J. Clin. Microbiol. 35, 257-260 (1997).

57 Pusch U, Effendy I, Schwarz RT, Azzouz N. Glycosylphosphatidylinositols synthesized by Trichophyton rubrum in a cell-free system. Mycoses 46, 104-113 (2003).

58 Marcon MJ, Powell DA. Human infections due to Malassezia spp. Clin. Microbiol. Rev. 5, 101-119 (1992).

59 Barros MB, de Almeida Paes R, Schubach AO. Sporothrix schenckii and sporotrichosis. Clin. Microbiol. Rev. 24, 633-654 (2011).

60 Lopes-Bezerra LM, Schubach A, Costa RO. Sporothrix schenckii and sporotrichosis. An. Acad. Bras. Cienc. 2, 293-308 (2006).

61 Lopes-Alves L, Travassos LR, Previato JO, MendonçaPreviato L. Novel antigenic determinants from peptidohramnomannans of Sporothrix Schenckii. Glycobiology 4, 281-288 (1994)

62 Ruiz-Baca E, Toriello C, Pérez-Torres A et al. Isolation and some properties of a glycoprotein of $70 \mathrm{kDa}(\mathrm{Gp} 70)$ from the cell wall of Sporothrix schenckii involved in fungal adherence to dermal extracellular matrix. Med. Mycol. 47, 185-196 (2009).

63 Bocca AL, Amaral AC, Teixeira MM et al. Paracoccidioidomycosis: eco-epidemiology, taxonomy and clinical and therapeutic issues. Future Microbiol. 8, 1177-1191 (2013).

64 Restrepo A, McEwen JG, Castaneda E. The habitat of Paracoccidioides brasiliensis: how far from solving the riddle? Med. Mycol. 39, 233-241 (2001).

65 Toledo MS, Suzuki E, Straus AH, Takahashi HK. Glycolipids from Paracoccidioides brasiliensis. Isolation of a galactofuranose-containing glycolipid reactive with sera of patients with paracoccidioidomycosis. J. Med. Vet. Mycol. 33, 247-251 (1995).

66 Suzuki E, Tanaka AK, Toledo MS, Levery SB, Takahashi HK, Straus AH. Trypanosomatid and fungal glycolipids and sphingolipids as infectivity factors and potential targets for development of new therapeutic strategies. Biochim. Biophys. Acta 1780, 362-369 (2008).

67 Hoog GS, Adelmann D, Ahmed AOA, van Belkum A. Phylogeny and typification of Madurella mycetomatis, with a comparison of other agents of eumycetoma. Mycoses 47, 121-130 (2004).
68 Brandt ME, Warnock DW. Epidemiology, clinical manifestations, and therapy of infections caused by dematiaceous fungi. J. Chemother. 15, 36-47 (2003).

69 Galili U, Shohet SB, Kobrin E et al. Man, apes, and old world monkeys differ from other mammals in the expression of alpha-galactosyl epitopes on nucleated cells. J. Biol. Chem. 263, 17755-17762 (1988).

70 San-Blas G, Prieto A, Bernabé M et al. Alpha-Galf $1 \rightarrow 6$-alpha-mannopyranoside side chains in Paracoccidioides brasiliensis cell wall are shared by members of the Onygenales, but not by galactomannans of other fungal genera. Med. Mycol. 43, 153-159 (2005).

71 Barr K, Laine RA, Lester RL. Carbohydrate structures of three novel phosphoinositol-containing sphingolipids from the yeast Histoplasma capsulatum. Biochemistry 23, 55895596 (1984).

72 Takayanagi T, Kimura A, Chiba S, Ajisaka K. Novel structures of $N$-linked high-mannose type oligosaccharides containing alpha-D-galactofuranosyl linkages in Aspergillus niger alphaD-glucosidase. Carbohydr. Res. 256, 149-158 (1994).

73 Dos Santos PO, Rodrigues AM, Fernandes GF, da Silva $\mathrm{SH}$, Burger E, de Camargo ZP. Immunodiagnosis of paracoccidioidomycosis due to Paracoccidioides brasiliensis using a latex test: detection of specific antibody anti-gp 43 and specific antigen gp43. PLoS Neg. Trop. Dis. 9(2), e0003516 (2015).

- A new rapid latex test for the detection of antigen gp43 and anti-gp43 in serum samples of $P$. brasiliensis mycosis patients.

74 Ferguson MAJ. GPI membrane anchors: isolation and analysis. In: Glycobiology: A Practical Approach. Fukuda M, Kobata A (Eds). Oxford University Press Oxford, UK, 349-383 (1993).

75 Ahrazem O, Gomez-Miranda B, Barasoaín I, Bernabé M, Leal JA. An acidic water-soluble cell wall polysaccharide: a chemotaxonomic marker for Fusarium and Gibberella. Mycol. Res. 104, 603-610 (2000).

76 Cattiaux L, Sendid B, Collot M et al. Synthetic biotinylated tetra $\beta(1 \rightarrow 5)$ galactofuranoside for in vitro aspergillosis diagnosis. Bioorg. Med. Chem. 19, 547-555 (2011).

- A tetrasaccharide, structural unit of a glycan from A. fumigatus was chemically synthesized. The conjugate with biotin could detect antibodies in sera from patients with aspergillosis.

77 van Heeswijk WAR, Visser HGJ, Vliegenthart JFG. Synthesis of 5-O- $\beta$-D-galactofuranosyl-D-galactofuranose. Carbohydr. Res. 59, 81-86 (1977).

78 Lederkremer RM, Marino C, Varela O. Convenient synthesis of 5-O-and 3,5-di- $O$-( $\beta$-D-galactofuranosyl)-Dgalactofuranose. Carbohydr. Res. 200, 227-235 (1990).

79 Pathak AK, Pathak V, Seitz L, et al. Studies on $(\beta 1 \rightarrow 5)$ and $(\beta 1 \rightarrow 6)$ linked octyl Gal $f$ disaccharides as substrates for mycobacterial galactosyltransferase activity. Bioorg. Med. Chem. 9, 3129-3143 (2001).

80 Gurjar MK, Reddy LK, Hotha S. Synthesis of oligosaccharides of motifs D and $\mathrm{E}$ of arabinogalactan present in Mycobacterium tuberculosis. J. Org. Chem. 66, 4657-4660 (2001). 
81 Sugawara F, Nakayama H, Ogawa T. Synthetic studies on derivatives of 5-O- $\beta$-D-galactofuranosyl-D-galactofuranose. Agric. Biol. Chem. 50, 1557-1561 (1986).

Veeneman GH, Notermans S, Liskamp RMJ, van der Marel GA, van Boom JH. Solid-phase synthesis of a naturally occurring $\beta$ - $(1 \rightarrow 5)$-linked D-galactofuranosyl heptamer containing the artificial linkage arm L-homoserine. Tetrahedron Lett. 28, 6695-6698 (1987).

Marino C, Varela O, Lederkremer RM. Synthesis of galactofuranose disaccharides of biological significance. Carbohydr. Res. 190, 65-76 (1989).

84 Euzen R, Ferrieres V, Plusquellec D. Synthesis of galactofuranose-containing disaccharides using thioimidoyltype donors. Carbohydr. Res. 341, 2759-2768 (2006).

85 Splain RA, Kiessling LL. Synthesis of galactofuranosebased acceptor substrates for the study of the carbohydrate polymerase GlfT2. Bioorg. Med. Chem. 18, 3753-3759 (2010).

Zhang G, Fu M, Ning J. An efficient and concise synthesis of a $\beta-(1 \rightarrow 6)$ linked D-galactofuranosyl hexasaccharide. Carbohydr. Res. 340, 155-159 (2005).

87 Gandolfi-Donadio L, Gallo-Rodriguez C, Lederkremer RM. Syntheses of $\beta-\mathrm{D}-\mathrm{Gal} f-(1 \rightarrow 6)-\beta-\mathrm{D}-\mathrm{Gal} f-(1 \rightarrow 5)-\mathrm{D}-\mathrm{Gal} f$ and $\beta$-D-Galf- $(1 \rightarrow 5)-\beta$-D-Galf-(1 $\rightarrow 6)$-D-Gal $f$, trisaccharide units in the galactan of Mycobacterium tuberculosis. J. Org. Chem. 68, 6928-6934 (2003).

Deng L-M, Liu X, Liang X-Y, Yang J-S. Regioselective glycosylation method using partially protected arabinoand galactofuranosyl thioglycosides as key glycosylating substrates and its application to one-pot synthesis of oligofuranoses. J. Org. Chem. 77, 3025-3037 (2012).

89 Marino C, Chiocconi A, Varela O, Lederkremer RM. The glycosylaldonolactone approach for the synthesisof B-D-Galf(1-3)-D-Man $p$ and 3-desoxi ß-D-xylohexofuranosyl(1-3)-D-Manp. Carbohydr. Res. 311, 183-189 (1998).

90 Baldoni L, Marino C. Synthetic tools for the characterization of galactofuranosyltransferases. Glycosylations via acylated glycosyl iodides. Carbohydr. Res. 374, 75-81 (2013).

91 Tsui DS, Gorin PA. Preparation of 8-methoxycarbonyloctyl glycosides of alpha-D-mannopyranose, 2- $O$-alphamannopyranosyl-alpha-D-mannopyranose, beta-Dgalactofuranose and 3-O-beta-D-galactofuranosyl-alphaD-mannopyranose. Carbohydr. Res. 156, 1-8 (1986).

92 Fu M, Zhang G, Ning J. First synthesis of the immunodominant $\beta$-galactofuranose-containing tetrasaccharide present in the cell wall of Aspergillus fumigatus. Carbohydr. Res. 340, 25-30 (2005).

93 Crick DC, Mahaprata S, Brennan PJ. Biosynthesis of the arabinogalactan-peptidoglycan complex of Mycobacterium tuberculosis. Glycobiology 11, 107-118 (2001).

94 Richards MR, Lowary TL. Chemistry and biology of galactofuranose-containing polysaccharides. Chembiochem 10, 1920-1938 (2009).

95 Peltier P, Euzen R, Daniellou R, Nugier-Chauvin C, Ferrières $\mathrm{V}$. Recent knowledge and innovations related to

hexofuranosides. Structure, synthesis and applications. Carbohydr. Res. 343, 1897-1923 (2008).

96 containing molecules. Design of galactofuranosyl acceptors. Chembiochem 15, 188-204 (2014).

97 Arasappan A, Fraser-Reid B. n-Pentenyl furanosides: synthesis and glycosidation reactions of some galacto derivatives. Tetrahedron Lett. 36, 7967-7070. (1995).

98 Marino C, Gandolfi-Donadío L, Gallo-Rodriguez C, Bai Y, Lederkremer RM. One-step syntheses of 1,2,3,5,6-penta$O$-benzoyl- $\alpha, \beta$-D-galactofuranose and 1,2,3,5-tetra- $O$ benzoyl- $\alpha, \beta-\mathrm{D}$-arabinofuranose. In: Carbohydrate Chemistry: Proven Methods. Kovac P (Ed.). Vol. 1, CRC Press. FL, USA, 231-238. (2011).

99 Schmidt RR, Jung K-H. In: Carbohydrate Chemistry and Biology. Vol. 2, Ernst B, Hart GW, Sinay P, (Eds). WileyVCH Verlag, Weinheim, Germany, (2000).

100 Gallo-Rodriguez C, Gandolfi L, Lederkremer RM. Synthesis of $\beta$-D-Galf- $(1 \rightarrow 3)$-D-GlcNAc by the tricloroacetamidate method and of $\beta$-D-Galf- $(1 \rightarrow 6)-\mathrm{D}$ GlcNAc by $\mathrm{SnCl}_{4}$-promoted glycosylation. Org. Lett. 1, 245-247 (1999).

101 Wu C-Y, Liang PH, Wong CH. New development of glycan arrays. Org. Biomol. Chem. 7, 2247-2254 (2009).

102 Johnson MA, Bundle DR. Designing a new antifungal glycoconjugate vaccine. Chem. Soc. Rev. 42, 4327-4344 (2013).

103 Pirofski L. Polysaccharides, mimotopes and vaccines for fungal and encapsulated pathogens. Trends Microbiol. 9, 445-451 (2001).

104 Stynen D, Goris A, Sarfati J, Latgé JP. A new sensitive sandwich enzyme-linked immunosorbent assay to detect galactofuran in patients with invasive aspergillosis. J. Clin. Microbiol. 33, 497-500 (1995).

105 Rohrlich P, Sarfati J, Mariani P et al. Prospective sandwich enzyme linked immunosorbent assay for serum galactomannan: early predictive value and clinical use in invasive aspergillosis. Pediatr. Infect. Dis. J. 15, 232-237 (1996).

106 Division of special pathogen and transplant products. Biomarker Qualification Microbiology Review Detection of Galactomannan in Serum by Platelia ${ }^{\mathrm{TM}}$ Aspergillus Enzymelinked Immunosorbent Assay (BioRad Laboratories and Sanofi Diagnostics) www.fda.gov/downloads/Drugs/

107 Chiodo F, Marradi M, Park J et al. Galactofuranose-coated gold nanoparticles elicit a pro-inflammatory response in human monocyte-derived dendritic cells and are recognized by DC-SIGN. ACS Chem Biol. 9, 383-389 (2014).

108 Mennink-Kersten MA, Donnelly JP, Verweij PE. Detection of circulating galactomannan for the diagnosis and management of invasive aspergillosis. Lancet Infect. Dis. 4, 349-357 (2004).

109 Maertens J, Theunissen K, Lodewyck T, Lagrou K, Van Eldere J. Advances in the serological diagnosis of invasive Aspergillus infections in patients with haematological disorders. Mycoses 50 (Suppl. 1), 2-17 (2007). 
110 Pasqualotto AC. (Ed.). Aspergillosis: From Diagnosis to Prevention. Springer, Berlin, Germany, 1027 (2010).

111 Nucci M, Carlesse F, Cappellano P et al. Earlier diagnosis of invasive fusariosis with Aspergillus serum galactomannan testing. PLoS ONE 9, e87784 (2014).

112 Tortorano AM, Esposto MC, Prigitano A et al. Cross-reactivity of Fusarium spp. in the Aspergillus galactomannan enzyme-linked immunosorbent assay. J. Clin. Microbiol. 50, 1051-1053 (2012).

113 Cummings JR, Jamison GR, Boudreaux JW, Howles MJ, Walsh TJ, Hayden RT. Cross-reactivity of non-Aspergillus fungal species in the Aspergillus galactomannan enzyme immunoassay. Diag. Micr. Infec. Dis. 59, 113-115 (2007).

114 Dalle F, Charles PE, Blanc K et al. Cryptococcus neoformans galactoxylomannan contains an epitope (s) that is crossreactive with Aspergillus galactomannan. J. Clin. Microbiol. 43, 2929-2931 (2005).

115 De Jesus M, Hackett E, Durkin M et al. Galactoxylomannan does not exhibit cross-reactivity in the Platelia Aspergillus enzyme immunoassay. Clin. Vaccine Immunol. 14, 624-627 (2007).

116 Vaishnav VV, Bacon BE, O’Neill M, Cherniak R. Structural characterization of the galactoxylomannan of Cryptococcus neoformans Cap67. Carbohydr. Res. 306, 315-330 (1998).
117 Heiss C, Skowyra ML, Liu H et al. Unusual galactofuranose modification of a capsule polysaccharide in the pathogenic yeast Cryptococcus neoformans. J. Biol. Chem. 288, 10994-11003 (2013).

118 every SB, Toledo MS, Straus AH, Takahashi HK. Structure elucidation of sphingolipids from the mycopathogen Paracoccidioides brasiliensis: an immunodominant betagalactofuranose residue is carried by a novel glycosylinositol phosphorylceramide antigen. Biochemistry 37, 8764-8775 (1998).

11 Carvalho KC, Vallejo MC, Camargo ZP, Puccia R. Use of recombinant gp 43 isoforms expressed in Pichia pastoris for diagnosis of paracoccidioidomycosis. Clin. Vaccine Immunol. 15, 622-629 (2008).

120 Puccia R, Travassos LR. The 43-kDa glycoprotein from the human pathogen Paracoccidioides brasiliensis and its deglycosylated form: excretion and susceptibility to proteolysis. Arch. Biochem. Biophys. 289, 298-302 (1991).

121 Wheat LJ, Hackett E, Durkin M et al. Histoplasmosisassociated cross-reactivity in the BioRad Platelia Aspergillus enzyme immunoassay. Clin. Vaccine Immunol. 14, 638-640 (2007). 